\title{
STAR FORMATION EFFICIENCIES AND STAR CLUSTER FORMATION
}

\author{
Uta Fritze - v. Alvensleben \\ Universitätssternwarte Göttingen \\ Geismarlandstr. 11, 37083 Göttingen, Germany \\ ufritze@uni-sw.gwdg.de
}

\begin{abstract}
Starbursts produce large numbers of Young Star Clusters (YSCs). Multi-color photometry in combination with a dedicated SED analysis tool allows to derive ages, metallicities, $\mathrm{E}_{\mathrm{B}-\mathrm{V}}$, and masses including $1 \sigma$ uncertainties for individual clusters and, hence, mass functions for YSC systems. The mass function, known to be Gaussian for old Globular Cluster (GC) systems, is still controversial for YSC systems. GC formation is expected in massive gas-rich spiral - spiral mergers because of their high global star formation efficiencies and observed in $\geq 1$ Gyr old merger remnants. Yet it has not been possible to identify young GCs among YSC populations. We suggest a compactness parameters involving masses and half-light radii of YSCs to investigate if young GCs are formed in starbursts and if the ratio of young GCs to more loosely bound star clusters depends on galaxy type, mass, burst strength, etc.
\end{abstract}

\section{Star Formation Efficiencies \& Star Cluster Formation}

Both burst strengths $b$ defined by the relative increase of the stellar mass in the course of starbursts, $b:=\frac{\Delta S_{\text {burst }}}{S_{\text {total }}}$, and Star Formation Efficiencies (SFEs) defined as the total stellar mass formed out of an available mass of gas, SFE := $\frac{\Delta S_{\text {burst }}}{G}$, are difficult to determine. Reasonable estimates are only possible in young post-starbursts. As long as a burst is active only lower limits can be given. Once a burst is over or if a burst lasts longer than the most massive stars' lifetimes, the amount of stars already died needs to be accounted for. For the stellar and gaseous masses before the burst can only be estimated on the basis of Hubble types, HI observations, etc. The strongest bursts are reported in mergers of massive gas-rich galaxies with total burst durations of the order of a few 100 Myr. Bursts in massive interacting galaxies are much stronger and last much longer than those in isolated dwarf galaxies. Blue Compact Dwarf galaxies (BCDs), e.g., feature bursts with durations of the order of a few Myr, $\mathrm{b} \ll 0.1, \mathrm{SFE} \leq 0.01$, and a trend of decreasing burst strengths 
for increasing total galaxy masses (including HI) (Krüger et al. 1995). Massive interacting galaxies feature bursts stronger and more efficient by one to two orders of magnitude, similar to the progenitors of E+A galaxies in clusters, ULIRGS, SCUBA galaxies, and optically identified starburst galaxies in the early universe. The post-burst spiral - spiral merger remnant NGC 7252 with two long, gas-rich tidal tails pointing at an age of $\lesssim 1 \mathrm{Gyr}$ after the onset of the strong interaction and its blue and radially constant colors and very strong Balmer absorption line spectrum must have experienced a very strong and global starburst increasing its stellar mass by as much as $\sim 40 \%$ between 600 and $1000 \mathrm{Myr}$ ago. Conservative estimates still lead to a very high SFE $\geq 30 \%$ (Fritze - v. Alvensleben \& Gerhard 1994). A large number of Star Clusters (SCs) formed throughout the main body, many of them apparently so strongly bound that they managed to survive for $500-900 \mathrm{Myr}$ the violent relaxation phase that restructured the remnant into a de Vaucouleurs profile (cf. Fritze - v. Alvensleben \& Burkert 1995 and Schweizer 2002 for a recent review). Most of these star clusters are young Globular Clusters (GCs) based on their ages, luminosities, and radii. How many clusters were already destroyed since the onset of the burst? An analogous system at a younger age is NGC 4038/39 where the two spiral disks just started overlapping. Its burst around the two nuclei, along the tidal structures, and - strongest - in the optically obscured disk - disk overlap region is in its initial stage. Thousands of bright Young Star Clusters (YSCs) are seen with luminosities ranging from those of individual red supergiant stars to $\mathrm{M}_{\mathrm{V}} \geq-15$. How many of these will survive for $\gg 1 \mathrm{Gyr}$ and become Globular Clusters (GCs)?

Hydrodynamic modelling shows that the formation of long-lived strongly bound GCs requires SFEs $\gg 10 \%$, originally thought to only occur in the early universe. In normal SF in spirals, irregulars, and starbursting isolated dwarfs like BCDs, GC formation should not be possible. In the high pressure ISM with its strong shocks in spiral - spiral mergers, however, GC formation is observed in reality and in high-resolution hydrodynamical models (Yuexing et al. 2004). Young and intermediate age GCs hence are tracers of high SFE periods in their parent galaxies. A number of very fundamental questions are still open at present: Does the amount of SF that goes into massive, compact, long-lived SCs scale with burst strength and/or (local/global) SFE? Or is there a threshold in SFE, below which only field stars and weakly bound, less massive $\mathrm{SCs}$ or $\mathrm{OB}$-associations can be formed that dissolve on timescales of $10^{8}$ $\mathrm{yr}$, and above which GCs can be formed or even become the dominant component? Does the same star and SC formation mechanism work in vastly different environments and scale over a huge dynamical range or are there two different modes of SF like "normal" and "violent"? SCs are seen to form in many environments from normal Irrs and spirals through dwarf starbursts, spiral mergers, and ULIRGs, constrained to nuclear regions (e.g. in ULIRGs), over their main 
body (e.g. NGC 4038/39), and all along tremendous tidal tails (e.g. Tadpole cf. de Grijs et al. 2003). The spatial extent of a starburst probably depends on the orbit and relative orientations of the interacting galaxies, on whether or not they had massive bulges and/or DM halos. Are all these YSC systems similar or systematically different in terms of masses, mass functions, sizes, compactness or degree of binding and, hence, survival times.

SCs are Simple Stellar Populations (SSPs) with all stars having the same age and chemical composition. Evolutionary synthesis models like GALEV describe the evolution of SCs over a Hubble time, from the youngest stages of 4 Myr all through the oldest GC ages $\geq 14$ Gyr for 5 different metallicities $-1.7 \leq[\mathrm{Fe} / \mathrm{H}] \leq+0.4$. The TP-AGB phase is very important for age-dating of SCs between $100 \mathrm{Myr}$ and a few Gyr on the basis of their V - I colors (cf.

Schulz et al. 2002). Gaseous emission in terms of lines and continuum for the respective metallicities makes important contributions to broad band fluxes and colors at young ages (Anders \& Fritze - v. Alvensleben 2003). Lick absorption indices significantly help disentangle ages and metallicities of older SCs (Lilly \& Fritze - v. Alvensleben in prep.). GALEV models yield the detailed spectral evolution of SCs from $90 \AA$ through $160 \mu \mathrm{m}$, luminosities, M/L-ratios, and colors in many filter systems (Johnson, HST, Washington, Stroemgren, ...) and can be retrieved from http://www.uni-sw.gwdg.de/ galev/ .

\section{Analysing Star Cluster Systems}

The time evolution of luminosities, colors, and M/L-ratios significantly depends on metallicity in a way that is different in different wavelengths regions. For young SC systems, like in NGC 4038/39, extinction is an important issue. Older starbursts, like in NGC 7252, are significantly less extincted. An ESO - ASTROVIRTEL project provides us with HST and VLT multi- $\lambda$ photometry for SC systems from young to old that have 4 and more passbands observed. A dedicated Spectral Energy Distribution (SED) analysis tool called AnalySED compares observed SC SEDs with an extensive grid of 117000 SSP model SEDs for 5 different metallicities, 1170 ages from 4 Myr through $14 \mathrm{Gyr}$, and 20 extinction values $0 \leq \mathrm{E}_{\mathrm{B}-\mathrm{V}} \leq 1$. We use Calzetti et al. 's (2000) starburst extinction law since internal extinction is only an issue in ongoing starbursts. A probability $\mathrm{p}(\mathrm{n}) \sim \exp \left(-\chi^{2}\right)$ is assigned to each model SED by a maximum likelihood estimator $\chi^{2}=\sum_{\lambda}\left(\mathrm{m}_{\lambda}^{\text {obs }}-\mathrm{m}_{\lambda}^{\text {model }}\right)^{2} / \sigma_{\text {obs }}^{2}$. The best fit model is the one with the highest probability. Probabilities are normalised to $\sum_{n} \mathrm{p}(\mathrm{n})=1$. Summing models with decreasing probabilities until $\sum_{\mathrm{n}} \mathrm{p}(\mathrm{n})=0.68$ provides $\pm 1 \sigma$ uncertainties for ages, metallicities, exticntion values, and masses of individual SCs (Anders et al. 2004a). Testing AnalySED with artificial SCs, we found that there are good and bad passband combinations, slightly depending on the ages and metallicities of the clusters, and we 
identified a combination of 4 passbands $\mathrm{U}, \mathrm{B}, \mathrm{V}$ or I, and $\mathrm{H}$ or $\mathrm{K}$ with observational photometric accuracy $\leq 0.2 \mathrm{mag}$ as optimal for YSC systems. We agree with the independent investigation by Cardiel et al. (2003), that at typical photometric accuracies broad band photometry with useful passband combinations is as powerful in disentangling ages and metallicities as is spectroscopy with typical S/N. The AnalySED tool is currently extended to also include Lick indices for analyses of intermediate-age and old GC systems (Lilly et al. , in prep., cf. Lilly's poster on the accompanying CD-ROM). In the dwarf starburst galaxy NGC 1569 we identify 169 YSCs on the ASTROVIRTEL images, the bulk of them with ages $\leq 25 \mathrm{Myr}$, low extinction and metallicities. Their masses are typically in the range from $10^{3}$ to $10^{4} \mathrm{M}_{\odot}$, only the two Super SCs have masses in the mass range of GCs (cf. Fig.1 and Anders et al. 2004b).

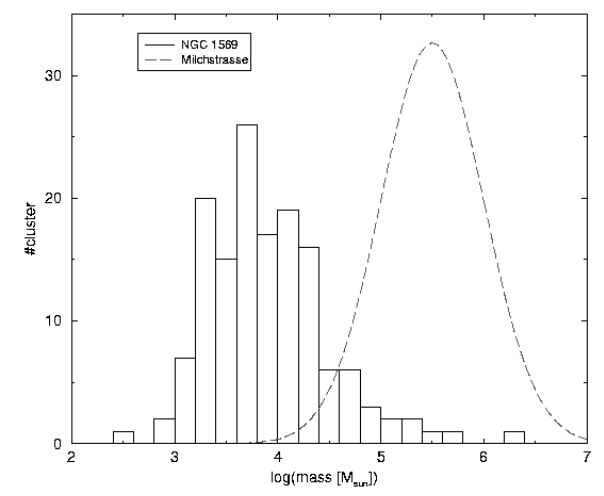

Figure 1. Distribution of YSC masses in NGC 1569 (histogram) as compared to the Milky Way GC mass function, normalised to the same number of clusters (Gaussian curve).

We conclude that the starburst in the dwarf galaxy NGC 1569 did not form many GCs, whereas the starburst in the spiral - spiral merger NGC 7252 did. Does SC formation produce a continuum in masses and binding energies or are there different modes of SC formation that respectively produce open and globular clusters? With increasing burst strength an increasing number of SCs is formed. Does the statistical effect of having a higher chance to have a more massive cluster within a larger sample explain the difference between cluster masses in NGC 1569 and NGC 7252? Probably not. Mass Functions (MFs) are power laws for open cluster systems and Gaussians for old GC systems. Initial MFs derived from models for survival and destruction of GCs in galactic potentials are controversial. Vesperini (2001) favors an initially Gaussian shape for GC MFs that is essentially conserved by the competing destructions of low-mass GCs through tidal disruption and of high-mass GC by dynamical friction. Gnedin \& Ostriker (1997) favor an initially power-law GC MF that 
is secularly transformed into a Gaussian by higher destruction rates for lower mass GCs. The MF of the young SC system in NGC 4038/39, that very probably comprises a mixture of OB-associations, open, and GCs, as derived from HST photometry, is also controversial. Whereas Zhang \& Fall (1999) derive a power-law MF using reddening-free $\mathrm{Q}_{1} \mathrm{Q}_{2}$ indices, we find a Gaussian $\mathrm{MF}$ (Fritze - v. Alvensleben 1998, 1999). Both approaches have their drawbacks. Zhang \& Fall excluded a significant number of clusters from the ambiguous age range in the $\mathrm{Q}_{1}-\mathrm{Q}_{2}$ - plot, we assumed a uniform average reddening in the WFPC1 UVI data. If we exclude the same SCs as Zhang \& Fall, we also find a power-law. The dust distribution in NGC 4038/39 is clearly patchy and a reanalysis of ASTROVIRTEL UBVIK data from HST WFPC2 and VLT ISAAC underway (Anders et al. in prep.). The shape of the MF need not correspond to the shape of the Luminosity Function (LF) for a young SC system, as age spread effects can distort the shape of the LF with respect to that of the underlying MF - to the point of transforming a Gaussian MF into a power-law LF up to the observational completeness limit. The key to survival or destruction is the strength of a SC's internal gravitational binding, as measured for Galactic GCs by their concentration parameters $c$. By definition, $c:=\log \frac{r_{t}}{r_{c}}$ involves the tidal and core radii. Very young clusters need not be tidally truncated yet and tidal radii could not be measured for the bulk of the YSCs on top of the bright galaxy background in NGC 4038/39 anyway. We therefore define the compactness of a young SC by the ratio between its mass and half-light radius (cf. Anders et al. in prep.), a robust quantity that can reliably be measured and is predicted by dynamical SC evolution models not to significantly change over a Hubble time. To this aim, we first have to improve upon the determination of $\mathrm{SC}$ radii by using appropriate aperture corrections. Improved SC radii, in turn, lead to improved SC photometry, and, hence, to improved photometric masses (cf. Poster by P. Anders on the accompanying CD-ROM).

\section{Conclusions and Perspective}

From the ages, masses, and radii of their SCs we know that major gas-rich mergers can form significant secondary populations of GCs in their strong and global starbursts. SFEs in mergers are higher by $1-2$ orders of magnitude than in normal SFing galaxies and (non-interacting) dwarf galaxy starbursts. Comparing good precision photometry in at least 4 reasonably chosen passbands (e.g. UBVK) to GALEV evolutionary synthesis models for SCs with given age, metallicity, extinction, and mass by means of a dedicated SED analysis tool (AnalySED) allows to reliably determine individual SC ages, metallicities, extinction values, and masses, including their respective $1 \sigma$ uncertainties. The first dwarf galaxy starburst analysed in detail this way shows only very few clusters with masses in the range of GCs among its $\sim 170$ YSCs. Clearly, 
both more major merger and dwarf galaxy starbursts need to be analysed in detail. Pixel-by-pixel analyses (de Grijs et al. 2003) or integrated field spectroscopy can provide burst strengths and SFEs. From a comparison with HST multi- $\lambda$ imaging of their YSC systems the relative ratios of SF going into field stars, short-lived open clusters, and long-lived GCs, respectively, can be determined. A key question is whether these quantities as well as the intrinsic properties of the YSCs, like masses and half-mass radii, depend on environment or not, in a smooth way or with some threshold. A comparison of starbursts in dwarf and massive, interacting and non-interacting starbursts should tell if SF and SC formation are universal processes or depend on environment. GC age and metallicity distributions will allow to trace back a galaxy's violent (star) formation history and constrain galaxy formation scenarios (Fritze v. Alvensleben 2004). This requires B through NIR photometry and medium resolution spectra to measure Lick indices. With only one observed color we cannot disentangle the age - metallicity degeneracy of intermediate-age and old stellar populations and see if more than one GC population is hidden in the red peak of many elliptical galaxies' bimodal color distributions.

\section{Acknowledgments}

I gratefully acknowledge travel support, in part from the DFG (FR 916/10-2) and in part from the organisers.

\section{References}

Anders, P., Fritze - v. Alvensleben, U., 2003, A\&A 401, 1063

Anders, P., Bissantz, N., Fritze - v. Alvensleben, U., de Grijs, R., 2004a, MN 347, 196

Anders, P., de Grijs, R., Fritze - v. Alvensleben, U., Bissantz, N., 2004b, MN 347, 17

Calzetti, D., Armus, L., Bohlin, R. C., Kinney, A. L., Koorneef, J., Storchi - Berrgmann, T., 2000, ApJ 533,682

Cardiel, N., Gorgas, J., Sánchez - Blázquez, P., Cenarro, A. J., Pedraz, S., Bruzual, G., Klement, J., 2003, A\&A 409, 511

Fritze - v. Alvensleben, U., 1998, A\&A 336, 83

Fritze - v. Alvensleben, U., 1999, A\&A 342, L25

Fritze - v. Alvensleben, U., 2004, A\&A 414, 515

Fritze - v. Alvensleben, U., Burkert, A., 1995, A\&A 300, 58

Fritze - v. Alvensleben, U., Gerhard, O. E., 1994, A\&A 285, 775

Gnedin, O. Y., Ostriker, J. P., 1997, ApJ 474, 223

de Grijs, R., Lee, J., Mora Herrera, C., Fritze - v. Alvensleben, U., Anders, P., 2003, New Astron. 8, 155

Krüger, H., Fritze - v. Alvensleben, U., Loose, H.-H., 1995, A\&A 303, 41

Schulz, J., Fritze - v. Alvensleben, U., Möller, C. S., Fricke, K. J., 2002, A\&A 392, 1

Schweizer, F., 2002, IAU Symp. 207, 630

Vesperini, E., 2001, MN 322, 247

Yuexing, L., MacLow, M.-M., Klessen, R. S., 2004, ApJ 614, L29

Zhang, Q., Fall, S. M., 1999, ApJ 527, L81 